\title{
An Antiatherogenic Role for Folic Acid in Experimental Diabetes
}

\author{
Madan S. Pote, John M. Noronha, ${ }^{*}$ and Velappan Kesavan** \\ Radiation Biology and Biochemistry Division, \\ Bhabha Atomic Research Centre, \\ Bombay 400 085, India
}

(Received January 27, 1995)

\begin{abstract}
Summary The effect of folic acid on lipoprotein lipase and various lipid fractions was studied in streptozotocin-induced diabetic rats. An enhanced plasma and hepatic lipoprotein lipase activity has been observed in folate supplemented diabetic animals. The proatherogenic triglycerides and very-low-density lipoprotein cholesterol +low-density lipoprotein cholesterol levels were found to be elevated in the diabetic groups, and folic acid supplementation lowered these levels. A significant increase in the antiatherogenic high-density lipoprotein cholesterol levels was observed in the diabetic rats on folate supplementation. The ratio of total cholesterol to high-density lipoprotein cholesterol of $3.93 \pm 1.56$ observed in the diabetic groups was lowered to $2.04 \pm 0.25$ on folate supplementation. These observations suggest a role for folic acid as an antiatherogenic agent in experimentally induced diabetes.
\end{abstract}

Key Words: diabetes, folic acid, lipoproteins, cholesterol, triglycerides

Although diabetes is a disorder of carbohydrate metabolism characterized by hyperglycemia, it is usually accompanied by abnormalities in lipid metabolism. Both insulin-dependent (Type I) and noninsulin-dependent (Type II) diabetes mellitus are frequently associated with abnormal lipid metabolism. Insulin has an influence on lipid metabolism, and the insulin-deficient diabetes results in a hyperlipidemia that eventually leads to atherosclerosis [1]. An increased incidence of hyperlipidemia and of atherosclerosis is frequently associated with diabetes [2]. The prevalence of hyperlipidemia is variable, depending on the type and severity of the diabetes, nutritional status, and age.

Besides lipids and lipoproteins, a number of nonlipid parameters have also

* Deceased on 25th January, 1995.

**To whom correspondence should be addressed. 
been recently implicated in atherogenesis, the prominent among them being certain vitamins, homocysteine, and some metal ions [3-8]. Among the vitamins, vitamin $\mathrm{A}$ and vitamin $\mathrm{C}$ deficiencies were reported to have a key role in the pathogenesis of hypertension [9]. A significant redistribution of cholesterol in the lipoproteins of persons administered high doses of vitamin $E$ has been demonstrated $[10,11]$.

Folic acid is implicated to have an antiatherogenic role in man and rats [12]. The elevated levels of proatherogenic TG, very-low-density lipoprotein cholesterol (VLDL-C), and low-density lipoprotein cholesterol (LDL-C) in alcohol-induced folate-deficient rats were significantly lowered on folate supplementation, indicating an antiatherogenic role for folic acid [13]. We have recently observed a functional folate deficiency created in streptozotocin-induced diabetic rats [14]. Polyglutamylfolates, which are the more active coenzymic forms of the vitamin, were depleted in large amounts as diabetes progressed, which might adversely affect all folate-dependent reactions [15]. As folic acid deficiency is shown to adversely influence the various lipoprotein cholesterol profiles [13], the functional folate deficiency observed in streptozotocin-induced diabetic rats [15] may also have a similar effect on lipoprotein metabolism. The present studies deal with the changes and the possible effect of folic acid supplementation on various lipoprotein profiles in streptozotocin-induced diabetic rats.

\section{MATERIALS AND METHODS}

Male Wistar strain rats weighing between $150-170 \mathrm{~g}$ were made diabetic by the single intraperitoneal administration of streptozotocin $(65 \mathrm{mg} / \mathrm{kg}$ body weight $)$. Rats developed diabetes, usually on the 4-5th day, which was confirmed by the value of the blood sugar level [14]. In order to evaluate the role of folic acid in influencing the lipoprotein profiles, the animals were divided into four groups: control, diabetic, control + folic acid, and diabetic + folic acid. Folic acid $(25 \mu \mathrm{g} /$ day/rat), wherever applicable, was intraperitoneally administered to rats consecutively for 25 days beginning on the 5 day after streptozotocin injection. The animals were then sacrificed $30 \mathrm{~min}$ following an i.p. administration of 25 I.U. heparin, which induces the release of lipase [12].

Blood from the portal vein was collected and incubated at $37^{\circ} \mathrm{C}$ for $30 \mathrm{~min}$, and the plasma was obtained by centrifugation at $1,000 \times \mathrm{g}$ for $15 \mathrm{~min}$ at room temperature in a clinical centrifuge.

A $10 \%$ liver homogenate was prepared in $0.05 \mathrm{M}$ phosphate buffer, $\mathrm{pH} 6.5$, and centrifuged at $800 \times g$ for $20 \mathrm{~min}$ at room temperature in clinical centrifuge. The resulting supernatant was used for the determination of hepatic lipoprotein lipase activity.

Determination of lipase activity. The lipase activity was determined by the turbidimetric assay by following the optical density changes in an olive oil emulsion used as substrate [16]. The reaction mixture for lipase determination contained olive oil emulsion $(3 \mathrm{ml})$ and $0.1 \mathrm{ml}$ enzyme source (plasma or liver) 
taken in a cuvette. The mixture was thoroughly mixed, and the temperature maintained at $37^{\circ} \mathrm{C}$ in a water bath. The lipase activity was determined by following turbidity changes every $1 \mathrm{~min}$ for $5 \mathrm{~min}$. The slope of the optical density curve obtained against the incubation time $(K)$ was used to calculate the enzyme activity. The lipase activity was expressed as the $K_{60}$ value [12].

Determination of total cholesterol (TC). Cholesterol was determined enzymatically with a commercial kit from A. Menarini Diagnostics, Italy. This method makes use of the enzymes cholesterol esterase, cholesterol oxidase, and peroxidase, with final product being spectrophotometrically measured at $500 \mathrm{~nm}$ [17]. The reaction was carried out as follows: To $10 \mu 1$ of plasma was added $1 \mathrm{ml}$ of the multi-enzyme reagent. The tubes were mixed and incubated in a water bath at $37^{\circ} \mathrm{C}$ for $10 \mathrm{~min}$, after which the optical density (O.D.) of the end product was read at $500 \mathrm{~nm}$ against a reagent blank. Ten microliters of distilled water was used as the sample blank, and $10 \mu \mathrm{l}$ of standard ( $200 \mathrm{mg} / \mathrm{dl}$ ) cholesterol (supplied along with the kit) served as the experimental standard. All assays were performed in duplicate. The assay was linear up to $500 \mathrm{mg} / \mathrm{dl}$ of cholesterol, and the color thus formed was stable for $30 \mathrm{~min}$ and was measured at $500 \mathrm{~nm}$ at room temperature.

Estimation of high-density lipoprotein cholesterol $(H D L-C)$. For estimation of HDL-C, $\beta$-lipoproteins including LDL-C and VLDL-C were selectively precipitated with phosphotungstic acid ( $13.9 \mathrm{~mm} /$ liter) and magnesium chloride (490 $\mathrm{mm} /$ liter) [18-20]. To $1 \mathrm{ml}$ of plasma taken in a fresh plastic tube, $100 \mu 1$ of phosphotungstic acid-magnesium chloride reagent (A. Menarini Diagnostics, Italy) was added. This was thoroughly mixed, and the mixture was then centrifuged at $400 \times g$ in a Sorwall RC $2 \mathrm{~B}$ centrifuge for $15 \mathrm{~min}$ at $4^{\circ} \mathrm{C}$. The supernatant, which contained HDL-C, was used to estimate cholesterol. The concentration of cholesterol thus obtained corresponds to the HDL-C level.

Estimation of plasma TG. Plasma TG levels were estimated by use of a commercial kit from A. Menarini Diagnostics, Italy, which makes use of the enzymes lipase, glycerokinase, glycerol-3-phosphate oxidase, and peroxidase [20, 21]. The reaction was carried out as follows: To $10 \mu 1$ of plasma was added $1 \mathrm{ml}$ of the reagent containing the above mentioned enzymes, and the tubes were mixed and incubated at $37^{\circ} \mathrm{C}$ for $10 \mathrm{~min}$ in a water bath. The O.D. of the final product was read at $500 \mathrm{~nm}$.

Estimation of plasma $L D L-C+V L D L-C . \quad$ LDL + VLDL cholesterol levels were calculated as the difference between TC and HDL-C levels.

Estimation of hepatic cholesterol and TG levels. The hepatic total cholesterol and TG levels were determined as follows: $1 \mathrm{~g}$ of the liver was homogenized in $2: 1$ chloroform : methanol, and the suspension was allowed to stand at room temperature overnight. The homogenate after filtration into a stoppered glass tube was added to $0.017 \% \mathrm{MgCl}_{2}$ and shaken well and decanted. The lower layer of the extract $(0.5 \mathrm{ml})$ was treated with $0.15 \mathrm{ml} \mathrm{33 \%} \mathrm{KOH}$ and $1.5 \mathrm{ml}$ of $95 \%$ ethanol and mixed thoroughly in glass-stoppered test tubes, which were then placed in a water bath for $30 \mathrm{~min}$. After the mixture had cooled, $5 \mathrm{ml}$ of hexane and $1.5 \mathrm{ml}$ of

Vol. 18, No. 3, 1995 
distilled water were added, and the mixture was shaken for 1 min. Aliquots $(1 \mathrm{ml})$ of the upper hexane layer were next withdrawn with a pipette, and the solvent was evaporated under nitrogen. The estimations of cholesterol and TG were then made as described above.

\section{RESULTS AND DISCUSSION}

Plasma lipoprotein lipase activity, measured as the $K_{60}$ value, showed no significant change in control and diabetic rats, the values being $0.80 \pm 0.15$ and $1.02 \pm 0.04$ (Table 1). However, on folate supplementation, the lipase activity was elevated by 2 fold $(2.17 \pm 0.08)$ in diabetic animals as compared with that of the respective control group $(1.02 \pm 0.04)$. The lipase activity after folic acid supplementation of the control group was found to be $1.10 \pm 0.07$. Although the hepatic lipase in control and diabetic animals showed (Table 1) similar activities $(0.30 \pm 0.03$ and $0.33 \pm 0.02$, respectively), a significant increase $(0.50 \pm 0.01, p<$ 0.01 ) in the total hepatic lipase activity was observed only in the folate-supplemented diabetic group.

The elevated lipase activity of folate administration in the diabetic rat might lead to an increased hydrolysis of TG from lipoproteins [22]. Povoa et al. [12] also observed similar increased activity of plasma lipase in the aorta and plasma of atherosclerotic rats following folic acid supplementation.

The hepatic profile of cholesterol and TG in the control and diabetic animals before and after folic acid supplementation is shown in Table 2. Although the

Table 1. Effect of folic acid administration on plasma and liver lipase activities in diabetic rats.

\begin{tabular}{lccccc}
\hline \multirow{2}{*}{ Conditions } & \multicolumn{3}{c}{ Lipase activity $\left(K_{60} \text { value }\right)^{*}$} \\
\cline { 2 - 3 } \cline { 5 - 6 } & - Folate & Plasma & & \multicolumn{2}{c}{ Liver } \\
\hline Control & $0.80 \pm 0.15$ & $1.10 \pm 0.07$ & & $0.30 \pm 0.03$ & $0.36 \pm 0.08$ \\
Diabetic & $1.02 \pm 0.04$ & $2.17 \pm 0.08^{* *}$ & & $0.33 \pm 0.02$ & $0.50 \pm 0.01^{* *}$ \\
\hline
\end{tabular}

The values are the mean \pm SEM for ten rats. ${ }^{*}$ The change in O.D. against incubation time extrapolated to $60 \mathrm{~min}$. ${ }^{* *}$ Significantly higher $(p<0.01)$ than the respective diabetic $(-)$ folate group.

Table 2. Effect of folic acid administration on liver cholesterol and triglyceride profiles in diabetic rats.

\begin{tabular}{llllll}
\hline \multirow{2}{*}{ Conditions } & \multicolumn{2}{c}{ Cholesterol $(\mathrm{mg} / \mathrm{g})$} & & \multicolumn{2}{c}{ Triglycerides $(\mathrm{mg} / \mathrm{g})$} \\
\cline { 2 - 3 } \cline { 5 - 6 } & - Folate & - Folate & & - Folate & + Folate \\
\hline Control & $1.3 \pm 0.1$ & $1.2 \pm 0.1$ & & $2.3 \pm 0.8$ & $2.5 \pm 0.2$ \\
Diabetic & $1.6 \pm 0.1$ & $1.2 \pm 0.1^{*}$ & & $4.6 \pm 0.5$ & $2.8 \pm 0.2^{*}$ \\
\hline
\end{tabular}

The values are the mean \pm SEM for ten rats. *Significantly lower $(p<0.02)$ than the respective diabetic $(-)$ folate group. 
Table 3. Plasma lipoprotein-cholesterol levels in control and diabetic animals before and after folate administration.

\begin{tabular}{lrccr}
\hline $\begin{array}{l}\text { Plasma lipoprotein } \\
\text { levels }\end{array}$ & Control & Diabetic & $\begin{array}{c}\text { Control } \\
\text { +folic acid }\end{array}$ & $\begin{array}{c}\text { Diabetic } \\
+ \text { folic acid }\end{array}$ \\
\hline HDL-C (mg/dl) & $63.79 \pm 8.53$ & $63.36 \pm 2.14$ & $58.18 \pm 10.66$ & $87.86 \pm 1.21^{*}$ \\
VLDL-C+LDL-C (mg/dl) & $91.66 \pm 5.50$ & $185.93 \pm 11.63$ & $107.50 \pm 6.83$ & $91.97 \pm 5.70^{* *}$ \\
TC (mg/dl) & $155.45 \pm 3.34$ & $249.29 \pm 2.90$ & $165.23 \pm 4.50$ & $179.83 \pm 2.42^{* *}$ \\
TG (mg/dl) & $94.96 \pm 4.50$ & $239.83 \pm 7.30$ & $111.67 \pm 2.60$ & $149.78 \pm 5.20^{* *}$ \\
TC/HDL-C (Ratio) & $2.43 \pm 0.50$ & $3.93 \pm 1.56$ & $2.83 \pm 0.60$ & $2.04 \pm 0.25^{\text {N.S. }}$ \\
\hline
\end{tabular}

The values are the mean \pm SEM for ten rats. ${ }^{*}$ Significantly higher $(p<0.001)$ than the respective value for the diabetic group. ${ }^{* *}$ Significantly lower $(p<0.001)$ than the respective

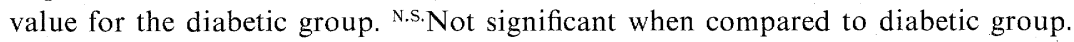

total hepatic cholesterol levels were not altered in either group, the TG levels in diabetic animals were almost 2 fold higher $(4.60 \pm 0.50 \mathrm{mg} / \mathrm{g}$ liver $)$ as compared with those of the control animals $(2.30 \pm 0.80 \mathrm{mg} / \mathrm{g})$. An increase in TG levels is known to occur in diabetes [23]. Administration of folic acid to these animals reduced the TG levels by $50 \%$ (to $2.80 \pm 0.20 \mathrm{mg} / \mathrm{g}$ ), which is comparable to the control values. However, no significant changes in the TG levels were observed after folate supplementation of control rats.

Various plasma lipoprotein-cholesterol levels before and after folate supplementation are shown in Table 3. Although the antiatherogenic HDL-C level in the diabetic group remained the same $(63.36 \pm 2.14 \mathrm{mg} / \mathrm{dl})$ as that of control + folic acid ones $(58.18 \pm 10.66)$, the supplementation of the vitamin to the diabetic animals resulted in an increase of these levels by $39 \%$ (to $87.86 \pm 1.21 \mathrm{mg} / \mathrm{dl}$ ).

On the other hand, the proatherogenic VLDL-C + LDL-C level in the diabetic rats was elevated 2 fold $(185.93 \pm 11.63 \mathrm{mg} / \mathrm{dl})$ as compared with that value for the control animals $(91.66 \pm 5.50 \mathrm{mg} / \mathrm{dl})$. However, this elevated VLDL-C + LDL-C level in the diabetic animals was lowered to a significant extent by the folic acid administration (to $91.97 \pm 5.70 \mathrm{mg} / \mathrm{dl}$ ). No gross change in the VLDL-C + LDL-C level was noticed in the control group on folate supplementation $(107.50 \pm 6.83$ $\mathrm{mg} / \mathrm{dl}$ ).

The total cholesterol level in the control animals was found to be $155.54 \pm$ $3.34 \mathrm{mg} / \mathrm{dl}$, while this level was elevated almost $60 \%$, to $249.29 \pm 2.90 \mathrm{mg} / \mathrm{dl}$, in the diabetic group. In folate-supplemented diabetic animals, the cholesterol levels had returned almost to normal $(179.83 \pm 2.42 \mathrm{mg} / \mathrm{dl})$ as compared with the control values.

The TG level (another of the group of proatherogenic risk factors), which was $94.96 \pm 4.50 \mathrm{mg} / \mathrm{dl}$ in the control rats, was elevated $2-3$ fold in the diabetic animals (to $239.83 \pm 7.30$ ). The folate-supplemented diabetic group showed a great reduction in the TG levels (to $149.78 \pm 5.20$ ). This drop occurred concomitantly with the elevation of plasma lipase activity after folate administration in the diabetic group (Table 1). The level of TG in the control + folic acid group, however, was $111.67 \pm$ $2.60 \mathrm{mg} / \mathrm{dl}$.

Vol. 18, No. 3, 1995 
A ratio of TC/HDL-C of greater than 5 has been considered to be an atherogenic risk factor in humans [24]. A comparison of the TC/HDL-C ratio in the present study showed $3.93 \pm 1.56$ for diabetic and $2.43 \pm 0.50$ for control animals (Table 3 ). The higher ratio of TC/HDL-C in the diabetes group suggests an increased risk of atherogenesis in these animals. However, on folate supplementation of the diabetic animals, the ratio significantly dropped to $2.04 \pm 0.25$.

A lipoprotein profile that is characterized by a high HDL-C level, low levels of LDL-C, TG, VLD-C + LDL-C, and a low TC/HDL-C ratio is considered as antiatherogenic [25]. In the present study folic acid was observed to have a role as an antiatherogenic agent in reducing the elevated atherogenic parameters. The possibility that the observed effect of folic acid on the modulation of lipoprotein profiles was caused by repression of the development of diabetes or by any direct action on lipid metabolism was ruled out, as the diabetic animals continued to have elevated blood glucose levels $(370.2 \pm 90 \mathrm{mg} \%$ as compared with $112.5 \pm 6$ $\mathrm{mg} \%$ for control rats). A similar antiatherogenic role for the vitamin was recently reported based on experiments on alcohol-fed folate-deficient animals conducted in our laboratory [13].

The mechanism by which folic acid lowers the levels of the proatherogenic factors is not well understood. Folic acid might act through its involvement in the methylation of homocysteine to methionine. Elevated plasma levels of homocysteine have been associated with an increased risk of atherosclerosis [26]. Homocysteine levels may be elevated in folic acid deficiency [27] or by the absence of any of the enzymes involved in the donation of a methyl group for the conversion of homocysteine to methionine [28]. A drastic depletion (95\%) of polyglutamylfolate derivatives, which are active coenzymic forms of the vitamin, was recently observed by us in streptozotocin-induced diabetic rats [13, 14], a depletion leading to a functional folate deficiency. This could result in a defective homocysteine to methionine conversion, leading to the accumulation of homocysteine. The high concentrations of homocysteine are known to injure the endothelium of the arterial wall and could cause enhanced detachment of endothelial cells, changes that could lead to atherogenesis [28]. Further, elevated plasma homocysteine levels are also known to be positively correlated with TC, TG, and LDL-apoB levels [28]. Lowering of elevated homocysteine levels reduces cardiovascular risks [29]. The clinical significance of folate in atherosclerosis observed here could lie in its ability to lower the elevated homocysteine level rather than to any direct association of the vitamin in the etiology of coronary heart disease [29].

\section{REFERENCES}

1. Gibbons, G.F. (1986): Hyperlipidaemia of diabetes. Clin. Sci., 71, 477-486.

2. Kraemer, F.B. (1987): Diabetes and lipoprotein receptors. Diabetes Metab. Rev., 3, 591-618.

3. Watanabe, J., Umeda, F., Wakasushi, H., and Ibayashi, H. (1984): Effect of vitamin E on platelet aggregation in diabetes mellitus. Thromb. Haemostasis, 51, 313-316. 
4. McCully, K.S. (1993): Chemical pathology of homocysteine. I. Atherogenesis. Ann. Clin. Lab. Sci., 23, 475-493.

5. Unauthored (1989): Homocysteine, folic acid and the prevention of vascular disease. Nutr. Rev., 47, 247-253.

6. Speich, M., Bousquet, B., and Nicolas, G. (1980): Concentrations of magnesium, calcium, potassium and sodium in human heart muscle after acute myocardial infarction. Clin. Chem., 26, 1662-1665.

7. Ebel, H. (1983): Role of magnesium in cardiac disease. J. Clin. Chem. Clin. Biochem., 21, $249-265$.

8. Tan, I.K., Chua, K.S., and Toh, A.K. (1992): Serum magnesium, copper and zinc concentrations in acute myocardial infarction. J. Clin. Lab. Anal., 6, 324-328.

9. McCarron, D.A., Morris, C.D., Henry, H.J., and Stanton, J.L. (1984): Blood pressure and nutrient intake in the United States. Science, 224, 1392-1398.

10. Hermann, W.J., Jr., Ward, K., and Faucett, J. (1983): The effect of tocopherol on highdensity lipoprotein cholesterol. Am. J. Clin. Pathol., 72, 848-852.

11. Barboriak, J.J., El Ghatti, A.Z., Shetty, K.R., and Kalbfleisch, J.H. (1982): Vitamin E supplements and plasma high-density lipoprotein cholesterol. Am. J. Clin. Pathol, 77, 371372.

12. Povoa, H., Marcondes, N., Fernandes, L., and Vicente, M.M. (1984): Folic acid and lipoprotein lipase from aorta and blood plasma of atherosclerotic rats. Biomed. Biochem. Acta, 43, 241-244.

13. Noronha, J.M., Kesavan, V., and Viswanathan, G. (1992): Antiatherogenic effect of folic acid in alcoholism. Pteridines, 3, 143-144.

14. Pote, M.S., Kesavan, V., and Noronha, J.M. (1994): Deranged folic acid metabolism in diabetes-induced rats. Indian J. Biochem. Biophys., 31, 68-72.

15. Kesavan, V., Pote, M.S., and Noronha, J.M. (1994): Folate metabolism in streptozocininduced diabetic rats. J. Clin. Biochem. Nutr., 16, 51-58.

16. Shihabi, Z.K., and Bishop, C. (1971): Simplified turbidimetric assay for lipase activity. Clin. Chem., 17, 1150-1153.

17. Allain, C.C., Poon, L.S., Chan, C.S.G., Richmond, W., and Fu, P.C. (1974): Enzymatic determination of total serum cholesterol. Clin. Chem., 20, 470-475.

18. Lopes-Virella, M.F., Stone, P., Ellis, S., and Colwell, J.A. (1977): Cholesterol determination in high density lipoproteins separated by three differential methods. Clin. Chem., 23, 882884.

19. Grove, T.H. (1979): Effect of reagent $\mathrm{pH}$ on determination of high density lipoprotein cholesterol by precipitation with sodium phosphotungstate-magnesium. Clin. Chem., 25, 560-564.

20. Bucolo, G., and David, H. (1973): Quantitative determination of serum triglycerides by the use of enzymes. Clin. Chem., 19, 476-482.

21. Megraw, R.E., Dunn, D.E., and Biggs, H.G. (1979): Manual and continuous-flow colorimetry of triglycerols by fully enzymatic method. Clin. Chem., 25, 273-278.

22. Nomura, T., Hagino, Y., Gotoh, M., Iguchi, A., and Sakamoto, N. (1984): The effects of streptozotocin diabetes on tissue specific lipase activities in the rat. Lipids, 19, 594-599.

23. Golay, A., Swislocki, A.L.M., Chen, Y.-D.I., and Reaven, G.M. (1983): Relationship between plasma free fatty acids concentration, endogenous glucose production and fasting hyperglycemia in normal and NIDDM individuals. Metabolism, 32, 971-976.

24. Gordon, T., Castelli, W.P., Hjortland, M.C., Kenneel, W.B., and Dawber, T.R. (1977): High density lipoprotein as a protective factor against coronary heart disease. The Framingham study. Am. J. Med., 62, 707-714.

25. McCully, K.S. (1983): Homocysteine theory of arteriosclerosis: Development and current status, in Atherosclerosis Reviews, Vol. 11, ed. by Gotto, A.M., Jr., and Paoletti, R., Raven Press, New York, pp. 157-246. 
26. Kang, S.S., Wong, P.W.K., and Norusis, M. (1987): Homocysteinemia due to folate deficiency. Metabolism, 36, 458-462.

27. Malinlinow, M.R. (1990): Hyperhomocyst(e)inemia. A common and easily reversible risk factor for occlusive atherosclerosis. Circulation, 81, 2004-2006.

28. Wilcken, D.E.L., Dudman, N.P.B., Tyrell, P.A., and Robertson, M.R. (1988): Folic acid lowers elevated plasma homocysteine in chronic renal insufficiency: Possible implications for vascular disease. Metabolism, 37, 697-701.

29. Pancharuniti, N., Lewis, C.A., Sauberlich, H.E., Perkins, L.L., Go, R.C.P., Alvarez, J.D., Maculso, M., Acton, R.T., Copeland, R.B., Cousins, A.L., Gore, T.B., Cornwell, P.E., and Roseman, J.M. (1994): Plasma homocyst(e)ine-folate and vitamin $\mathrm{B}_{12}$ concentrations and risk for early onset of coronary artery-disease. Am. J. Clin., 59, 940-948. 